The external glands empty their lymph into the internal jugular group, which in their turn drain one into the other as they descend the neck, finally emptying into the jugular lymph trunk. This lymph trunk empties into the junction of the internal jugular and subclavian veins, and on the left side of the neck is occasionally joined by the thoracic duct just before it enters the vein.

The supra-clavicular glands occupy the subclavian triangle, and the possibility of connection between this group and the deep cervical glands of the neck is the chief contention in the question of infection from the throat reaching the pleura. G. B. Wood, whom we are quoting, argues against such direct connection, though we are not sure but that his experiments might not bear an exactly reverse interpretation. The point is not settled, but it is one that is so important that all should be familiar with the work that is being done upon it, even though the results so far seem indecisive. The supra-clavicular glands receive lymphatics from the posterior part of the hairy scalp and from the muscles of the neck; also from the skin of the arm and chest, and an efferent branch from the humeral chain $\mathrm{cf}$ the axillary group. Their efferent duct generally empties into the jugular trunk just before it enters the vein.

Wood suggests that the injection of the supraclavicular gland in one of his cases took place against the normal direction of current, owing to the node situated in the venous notch obstructing the downward flow, so that the fluid on entering this node passed as readily up the efferent of the supraclavicular gland as into the jugular trunk. It is by no means clear, however, what right one has to make any such assumption, rather than to allow that materials may naturally pass from the upper cervical lymphatics directly into the supra-clavicular glands, and thus into close relationship with the pleura. It is true that the supra-clavicular glands are themselves a little distance from the apical pleura, but they are connected with the node occupying the venous notch, and this is separated from the pleura only by the carotid artery and its sheath. Enlargement of this node sufficient to cause displacement either anteriorly or posteriorly would bring its capsule into almost direct contact with the pleura.

It is interesting to note the condition of the supraclavicular glands as found at autopsies upon phthisical patients. Out of seventeen consecutive cases tuberculous foci were found microscopically in these glands in fifteen. This is a very high percentage, and it makes one wonder whether the tubercle bacilli got into the glands after the lungs had first been affected, or whether the organisms had got into the glands first and thence had infected the lung. It is quite true that patients who suffer from extensive tuberculosis of the cervical glands in early life seem particularly immune to phthisis; it is also quite true that there was no obvious affection of the supra-clavicular glands during life in the above fifteen cases in which tubercles were found in them microscopically after death. It may be, therefore, that when the glands catch up the bacilli and become obviously caseous, extension is being arrested, and that there is another type of tuberculous lymphadenitis in which the organisms are allowed to extend along the lymphatic chain without causing clinical signs of any lymphaticinfection at all. It is a remarkable fact that the supra-clavicular lymphatic glands exhibit tuberculous foci in such a large number of phthisical cases. The subject of the path of infection in phthisis is one upon which views that seemed absolutely stable a few years ago are now looked upon as very far from certain. The view that phthisis is due to tubercle bacilli reaching the lungs viâ the bronchioles is being boldly challenged. Lymphatic infection from the upper respiratory channels, and thence an extension to the lung from the supraclavicular or closely related lymph nodes would explain very prettily why phthisis always begins near the apex of the lung. The subject is one upon which a great deal of research work could comparatively easily be done. An accumulation of evidence upon the microscopical characters of the different groups of lymphatic glands in the neck, thorax, and abdomen in fatal cases of phthisis might lead to very important discoveries.

\title{
THYROID TREATMENT OF PSORIASIS.
}

Psoriasis is a disease which very frequently gives way for the time being before the internal administration of arsenic and the external application of ointments containing coal-tar products or chrysarobin. The two points to which most particular attention needs to be directed for the successful treatment of the case are, first, that the dose of liquor arsenicalis should be increased by 1 minim weekly until as much as 10 minims or more are being taken three times a day; and, secondly, that the external application must be mild in proportion to the acuteness of the eruption, and strong only in chronic cases. Neglect to observe the latter rule sometimes results in a serious dermatitis supervening on the psoriasis.

There remain certain cases, however, in which the above routine treatment fails to cure even temporarily, and then one is only too glad of any fresh suggestions. One such is the administration of five grains of thyroid extract three times a day, as re- commended by Dr. Byrom Bramwell in his Clinical Studies. In some cases, without any external medication, the oral exhibition of thyroid extract is followed by rapid disappearance of the rash. Dr. Bramwell gives it as his opinion that although it will not prevent relapses any better than any other form of treatment does, in obstinate cases of psoriasis thyroid extract should always be tried, the effects being carefully watched in case of the development of untoward symptoms which might prevent its being continued.

The results of the treatment, extending over a series of cases, may be summarised as follows: (1) that in some cases - the exception rather than the rule-brilliant and remarkable results are obtained, small doses curing the condition rapidly; (2) that in some cases, if the remedy is given persistently, improvement, and, in a considerable number of cases, complete temporary cure, results; and (3) that in other cases there is little or no improvement. 